\title{
Blind I/Q Imbalance Compensation for M-QAM Optical Coherent Systems based on Pseudo-rotation
}

\author{
Ti Nguyen-Ti ${ }^{1}$, Matthieu Gautier ${ }^{1}$, Pascal Scalart ${ }^{1}$, Olivier Berder ${ }^{1}$, Trung-Hien Nguyen ${ }^{2}$, Faycal Ait Aoudia ${ }^{1}$ \\ ${ }^{1}$ University of Rennes 1, IRISA Laboratory, France \\ ${ }^{2}$ University of Rennes 1, FOTON Laboratory, CNRS, France
}

\begin{abstract}
This paper addresses the problem of Inphase/Quadrature (I/Q) imbalance sensitivity of communication systems when it occurs at both Transmitter (TX) and Receiver (RX) sides of an optical coherent system. A novel blind technique is proposed based on the pseudo-rotation of the M-QAM constellation. The pseudo-rotation based compensator is a generic approach, because it does not depend on the modulation order and the level of imbalance. To implement the proposed compensation in practical systems, two algorithms are proposed: Recursive Pseudo-Rotation (RPR) that achieves the performance of the ideal compensator and LRPR, a Low complexity version of RPR. Monte Carlo simulation results show that the proposed compensator outperforms state-of-the-art algorithms for an Additive White Gaussian Noise (AWGN) and the complexity/performance trade-off is also discussed. The efficiency of the proposed method is experimentally validated with a 10 Gbaud QPSK optical system showing its operation in the presence of Inter-Symbol Interference (ISI).
\end{abstract}

Index Terms-I/Q imbalance, optical coherent system, blind compensation, $M$-QAM

\section{INTRODUCTION}

M-QAM modulations combined with coherent detection becomes now a promising candidate for the implementation of next generation optical transmission systems. This is made possible thanks to technical progress in photonic integrated circuits allowing the fabrication of optical circuits for M-QAM signal generation [1]. Despite the good performance of these circuits, amplitude and phase mismatches between the MQAM in-phase and quadrature components are still important due to the non-linear gain of the electrical amplifiers, the phase shifts in optical waveguides and the cable lengths or circuit paths on printed boards. It is generally referred to as the I/Q imbalance problem which may be burdensome if not compensated [2].

In the effort of decreasing the effect of I/Q imbalance, two types of methods have been established: pilot-assisted and blind techniques. In pilot-assisted techniques [3][4][5], training data are used to estimate the imbalance parameters. The challenges for these techniques are to lower the amount of training data and to achieve a good synchronization before the compensation. To tackle these challenges, the blind compensation techniques are good candidates as they do not require any pilot data and thus, the synchronization requirement is also more flexible than for pilot-assisted techniques. Many blind I/Q imbalance compensators have been proposed such as blind Least Mean Square (LMS), blind recursive least square [6], I/Q imbalance compensation filter [7], Gram-Schmidt
Orthogonalization Procedure (GSOP) [8] and Blind Source Separation (BSS) [9][10].

The promising GSOP and BSS compensators both use a two-steps process: a whitening step to eliminate the mutual information between the I and Q components and a rotation step to compensate the phase offset. Considering these two steps separately is not efficient because of the difficulty of tracking the phase.

Therefore, in this paper, a novel blind I/Q imbalance compensation is proposed based on a pseudo-rotation technique. Instead of solving the phase offset separately, the proposed compensator will jointly perform the whitening and rotation steps by using a pseudo-rotation with one dedicated angle per I and Q component.

The contributions of this paper are:

- A novel blind technique for compensating at receiver side both TX and RX imbalances using a pseudo-rotation matrix,

- Two practical implementations of the proposed compensators, one achieving the performance of the ideal compensation and one with a low complexity,

- A fair comparison with two state-of-the-art techniques, namely GSOP and BSS compensators,

- An experimental validation in a full optical communication system demonstrating that the proposed technique is not disrupted by ISI.

The remainder of the paper is organized as follows. Section II gives a theoretical analysis of the I/Q imbalance by deriving error probabilities and computing the ideal compensator. The state-of-the-art methods GSOP and BSS are also introduced in this section. The proposed method is presented in Section III, with first the theoretical analysis and then practical implementations. The performance results are given in Section IV as well as the experimental validation. Finally, Section VI concludes this paper and discusses future works.

\section{Theoretical ANALYSis of I/Q IMbalance AND COMPENSATION}

Throughout this paper, the notations $\mathrm{E}[],.(.)^{*},(.)^{T}$, and $(.)^{H}$ are used to denote statistical expectation, conjugate, transpose, and Hermitian transpose, respectively, and the notation for the complex envelope of a modulated signal is denoted $x=x_{I}+j x_{Q}$, where $x_{I}, x_{Q}$ represent its in-phase and quadrature components, respectively. $M$ being the modulation order, M-QAM are considered such as $x_{I}, x_{Q} \in \mathcal{B}$ with 


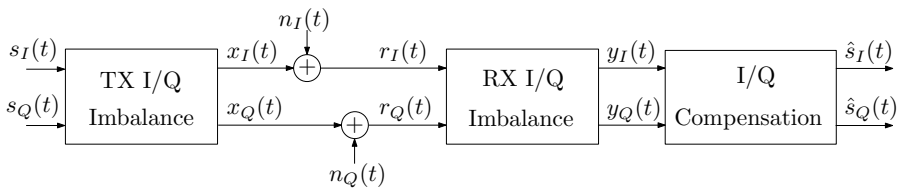

Fig. 1: Block diagram of the communication system with TX and RX I/Q imbalances and RX compensation.

$\mathcal{B}=\{(-\sqrt{M}+1) d ;(-\sqrt{M}+3) d ; \cdots ;-d ; d ; \cdots ;(\sqrt{M}-$ $3) d ;(\sqrt{M}-1) d\}$. The parameter $d$ is used to characterize the transmitted energy [11]:

$$
d=\sqrt{\frac{3 E_{s}}{2(M-1)}}=\sqrt{\frac{3 \log _{2}(M) E_{b}}{2(M-1)}},
$$

where $E_{b}, E_{s}$ are the bit and symbol energies, respectively.

\section{A. I/Q imbalance model}

Assuming (without loss of generality) that the I/Q imbalance only appears on the Q branch in the asymmetrical model [2], it can be characterized by 4 parameters: $g_{T}, \varphi_{T}, g_{R}, \varphi_{R}$ which correspond to the gain and phase imbalances at TX and RX, respectively. The equivalent baseband representation of the communication system is given in Fig. 1.

By noting $s(t)=s_{I}(t)+j s_{Q}(t)$ the unimpaired signal or the source signal and $x(t)=x_{I}(t)+j x_{Q}(t)$ the transmitted signal, the relation between $s(t)$ and $x(t)$ is expressed as:

$$
\left(\begin{array}{c}
x_{I} \\
x_{Q}
\end{array}\right)=\left(\begin{array}{rr}
1 & -g_{T} \sin \left(\varphi_{T}\right) \\
0 & g_{T} \cos \left(\varphi_{T}\right)
\end{array}\right)\left(\begin{array}{c}
s_{I} \\
s_{Q}
\end{array}\right)=\mathbf{B}\left(\begin{array}{c}
s_{I} \\
s_{Q}
\end{array}\right),
$$

where $\mathbf{B}$ is defined as the TX imbalance matrix.

Assuming that the transmission channel is modeled as an AWGN noise $n(t)=n_{I}(t)+j n_{Q}(t)$ with zero mean and variance $\sigma^{2}=N_{0}$ (both real and imaginary parts having a double-sided power spectral density equal to $N_{0} / 2$ ). The relation between the transmitted signal and the received signal $r(t)=r_{I}(t)+j r_{Q}(t)$ is:

$$
r(t)=x(t)+n(t) .
$$

At RX side, the I/Q imbalance model is inferred by:

$$
\left(\begin{array}{c}
y_{I} \\
y_{Q}
\end{array}\right)=\left(\begin{array}{cc}
1 & 0 \\
-g_{R} \sin \left(\varphi_{R}\right) & g_{R} \cos \left(\varphi_{R}\right)
\end{array}\right)\left(\begin{array}{c}
r_{I} \\
r_{Q}
\end{array}\right)=\mathbf{D}\left(\begin{array}{c}
r_{I} \\
r_{Q}
\end{array}\right),
$$

where $y(t)=y_{I}(t)+j y_{Q}(t)$ is the signal that needs to be compensated, and $\mathbf{D}$ the RX imbalance matrix.

\section{B. Ideal I/Q imbalance compensation}

The easiest method to eliminate the influence of I/Q imbalance is to do the inverse process. Therefore, the ideal I/Q imbalance compensator $\mathbf{B}_{d}$ at $\mathrm{Rx}$ side can be described as $\mathbf{B}_{d}=\mathbf{B}^{-1} \mathbf{D}^{-1}$. It is formulated by:

$$
\mathbf{B}_{d}=\left(\begin{array}{cc}
\frac{\cos \left(\varphi_{R}-\varphi_{T}\right)}{\cos \left(\varphi_{T} \cos \left(\varphi_{R}\right)\right.} & \frac{\tan \left(\varphi_{T}\right)}{g_{R} \cos \left(\varphi_{R}\right)} \\
\frac{\tan \left(\varphi_{R}\right)}{g_{T} \cos \left(\varphi_{T}\right)} & \frac{1}{g_{T} g_{R} \cos \left(\varphi_{T}\right) \cos \left(\varphi_{R}\right)}
\end{array}\right) .
$$

The output signal of such a compensator $\hat{s}(t)=\hat{s}_{I}+j \hat{s}_{Q}$ is given by:

$$
\left(\begin{array}{c}
\hat{s}_{I} \\
\hat{s}_{Q}
\end{array}\right)=\mathbf{B}_{d}\left(\begin{array}{c}
y_{I} \\
y_{Q}
\end{array}\right)=\left(\begin{array}{c}
s_{I} \\
s_{Q}
\end{array}\right)+\mathbf{B}^{-1}\left(\begin{array}{c}
n_{I} \\
n_{Q}
\end{array}\right) .
$$

This ideal compensation brings a strong improvement to the system performance. Unfortunately, the four imbalance parameters $g_{T}, g_{R}, \varphi_{T}, \varphi_{R}$ are unknown in practice and the compensator must be estimated using pilot-based or blind techniques. Blind solutions are considered in this work and two of most efficient compensators are introduced in the next part.

\section{State of the art of blind compensation methods}

[8] and [10] propose to split the compensation matrix $\mathbf{B}_{d}$ as the product of a whitening matrix $\mathbf{R}_{b}$ and a rotation matrix $\mathbf{R}_{r}$ :

$$
\mathbf{B}_{d}=\mathbf{R}_{r} \mathbf{R}_{b} .
$$

1) Whitening matrix: Two main algorithms are usually used for estimating this matrix: GSOP [8] is based on orthonormalising properties in the Euclidean space, and BSS [10] applies a serial update rule that satisfies the whitening condition.

In GSOP, the estimated whitening matrix is formulated as:

$$
\left(\hat{\mathbf{R}}_{b}\right)_{G S O P}=\frac{1}{\sigma_{I}}\left(\begin{array}{cc}
1 & 0 \\
\frac{\lambda}{\sigma_{I} \sigma_{Q^{\prime}}} & \frac{\sigma_{I}}{\sigma_{Q^{\prime}}}
\end{array}\right),
$$

where $\lambda=\mathrm{E}\left[y_{I}(t) y_{Q}(t)\right]$ is the correlation coefficient, $\sigma_{I}^{2}=\mathrm{E}\left[x_{I}^{2}(t)\right]$ is the power of the I component and $\sigma_{Q^{\prime}}^{2}=\mathrm{E}\left[\left(y_{Q}(t)-\lambda \frac{y_{I}(t)}{\sigma_{I}^{2}}\right)^{2}\right]$.

In BSS, the whitening matrix at time $t$ is formulated as:

$\left(\hat{\mathbf{R}}_{b}\right)_{B S S}(t+1)=\left(\hat{\mathbf{R}}_{b}\right)_{B S S}(t)-\mu\left[\mathbf{z}(t) \mathbf{z}^{H}(t)-\mathbf{I}\right]\left(\hat{\mathbf{R}}_{b}\right)_{B S S}(t)$,

where $\mu$ is the step size, $\mathbf{y}(t)=\left[y(t) y^{*}(t)\right]^{T}$, $\mathbf{z}(t)=\left[\begin{array}{ll}z(t) & z^{*}(t)\end{array}\right]^{T}=\left(\hat{\mathbf{R}}_{b}\right)_{B S S}(t) \mathbf{y}(t)$ and $\mathbf{I}$ the identity matrix.

2) Rotation matrix: $\mathbf{R}_{r}$ is described as:

$$
\mathbf{R}_{r}=\left(\begin{array}{cc}
\cos (\alpha) & \sin (\alpha) \\
-\sin (\alpha) & \cos (\alpha)
\end{array}\right)
$$

The value of the phase offset $\alpha$ can be estimated using a second order Digital Phase Locked Loop (DPLL) [12].

These two methods perform separately the whitening and the rotation steps thus resulting in a sub-optimal approach. Therefore, in this paper, we propose a joint approach based on the pseudo-rotation technique. 
TABLE I: Theoretical boundaries: the 4 regions are separated by the I/Q plane of the source signal $s(t)$.

\begin{tabular}{|c|c|c|c|c|}
\hline & Region $\mathcal{R}_{1}$ & Region $\mathcal{R}_{2}$ & Region $\mathcal{R}_{3}$ & Region $\mathcal{R}_{4}$ \\
\hline$s_{I}(t)$ & + & - & - & + \\
\hline$s_{Q}(t)$ & + & + & - & - \\
\hline
\end{tabular}

\section{PSEUdo-Rotation COMPENSATOR}

\section{A. Theoretical analysis of pseudo-rotation compensator}

In the proposed technique, the difficult problem related to the phase is addressed by using two different angles (one per I/Q component) to perform both the whitening and the rotation operations. A gain control is later applied on each component. Consequently, the ideal I/Q imbalance compensator can be decomposed as the product of a gain matrix $\mathbf{R}_{g}$ and a pseudorotation matrix $\mathbf{R}_{p}$ as follows:

$$
\mathbf{B}_{d}=\mathbf{R}_{g} \mathbf{R}_{p} .
$$

The pseudo-rotation matrix rotates complex points with two different angles versus two axes as shown in Fig. 2. It is formulated as:

$$
\mathbf{R}_{p}=\left(\begin{array}{rr}
\cos \left(\theta_{I}\right) & -\sin \left(\theta_{I}\right) \\
\sin \left(\theta_{Q}\right) & \cos \left(\theta_{Q}\right)
\end{array}\right) .
$$

We denote $z(t)=z_{I}(t)+j z_{Q}(t)$ the signal processed by $\mathbf{R}_{p}$ such as:

$$
\left[\begin{array}{ll}
z_{I} & z_{Q}
\end{array}\right]^{T}=\mathbf{R}_{p}\left[\begin{array}{ll}
y_{I} & y_{Q}
\end{array}\right]^{T} .
$$

Considering that the source symbols $s(t)=s_{I}(t)+j s_{Q}(t)$ can be separated into 4 sets as described in Table I, the TX/RX phase imbalances are totally compensated when the following conditions are satisfied:

$$
\left\{\begin{array}{l}
\mathrm{E}\left[z_{I}(t)\right]_{1}=\mathrm{E}\left[z_{I}(t)\right]_{4} \\
\mathrm{E}\left[z_{Q}(t)\right]_{1}=\mathrm{E}\left[z_{Q}(t)\right]_{2}
\end{array},\right.
$$

where $\mathrm{E}\left[z_{I}(t)\right]_{l}$ and $\mathrm{E}\left[z_{Q}(t)\right]_{l}$ are the mean of the inphase and quadrature components of $z(t)$ in region $\mathcal{R}_{l}$ for $l \in\{1,2,3,4\}$.

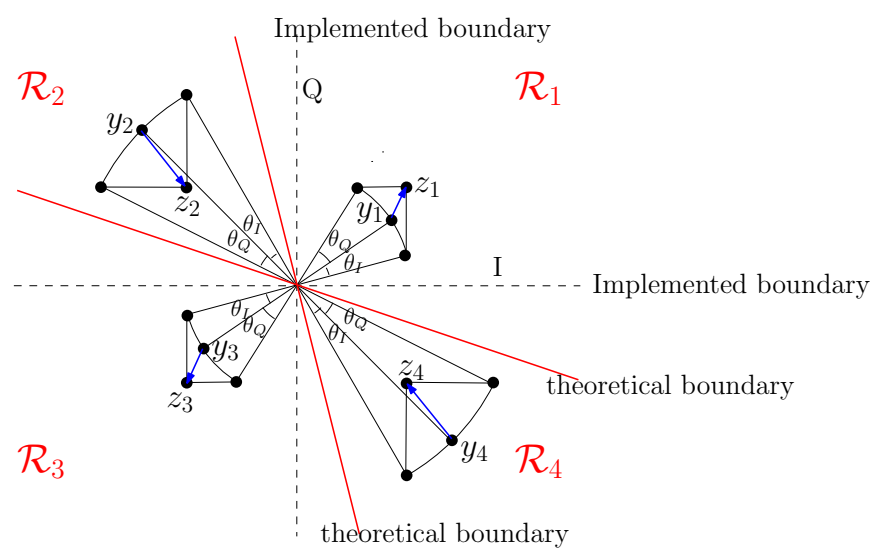

Fig. 2: Representation of pseudo-rotation transformation of imbalanced signal $y(t)$ on I/Q plane.
TABLE II: Implemented boundaries: the 4 regions are separated by the I/Q plane of the imbalanced signal $y(t)$.

\begin{tabular}{|c|c|c|c|c|}
\hline & Region $\mathcal{R}_{1}$ & Region $\mathcal{R}_{2}$ & Region $\mathcal{R}_{3}$ & Region $\mathcal{R}_{4}$ \\
\hline$y_{I}(t)$ & + & - & - & + \\
\hline$y_{Q}(t)$ & + & + & - & - \\
\hline
\end{tabular}

After rotation, the output signal $z(t)$ should be symmetrical across I and Q axes, so the pseudo-rotation angles satisfy :

$$
\begin{aligned}
\tan \left(\theta_{I}\right) & =\frac{\mathrm{E}\left[y_{I}(t)\right]_{4}-\mathrm{E}\left[y_{I}(t)\right]_{1}}{\mathrm{E}\left[y_{Q}(t)\right]_{4}-\mathrm{E}\left[y_{Q}(t)\right]_{1}}=-\frac{\sin \left(\varphi_{T}\right)}{g_{R} \cos \left(\varphi_{R}-\varphi_{T}\right)}(15) \\
\tan \left(\theta_{Q}\right) & =-\frac{\mathrm{E}\left[y_{Q}(t)\right]_{1}-\mathrm{E}\left[y_{Q}(t)\right]_{2}}{\mathrm{E}\left[y_{I}(t)\right]_{1}-\mathrm{E}\left[y_{I}(t)\right]_{2}}=g_{R} \sin \left(\varphi_{R}\right),
\end{aligned}
$$

where $\mathrm{E}\left[y_{I}(t)\right]_{l}, \mathrm{E}\left[y_{Q}(t)\right]_{l}$ are the mean of the in-phase and quadrature components of $y(t)$ in region $\mathcal{R}_{l}$ for $l \in\{1,2,3,4\}$ such as:

$$
\mathrm{E}\left[y_{I}(t)\right]_{l}=\mathrm{E}\left[y_{I}(t) \mid s(t) \in \mathcal{R}_{l}\right] .
$$

The transformation from the imbalanced signal to the pseudo-rotated signal is described in Fig. 2, where the 8 mean points $\mathrm{E}[y(t)]_{l}, \mathrm{E}[z(t)]_{l}$ are represented by $y_{l}$ and $z_{l}$ respectively, for $l \in\{1,2,3,4\}$.

The gain matrix $\mathbf{R}_{g}$ will later be easily computed by imposing equal absolute values of the 4 new means in both I and Q branches. Thus, it can be expressed as:

$$
\begin{aligned}
\mathbf{R}_{g} & =\left(\begin{array}{cc}
\frac{d \sqrt{M}}{2 \mathrm{E}\left[z_{I}(t) \mid s(t) \in \mathcal{R}_{1}\right]} & 0 \\
0 & \frac{d \sqrt{M}}{2 \mathrm{E}\left[z_{Q}(t) \mid s(t) \in \mathcal{R}_{1}\right]}
\end{array}\right) \\
& =\frac{1}{\cos \left(\varphi_{T}\right) \cos \left(\varphi_{R}\right)}\left(\begin{array}{cc}
\frac{\cos \left(\varphi_{R}-\varphi_{T}\right)}{\cos \left(\theta_{I}\right)} & 0 \\
0 & \frac{1}{\cos \left(\theta_{Q}\right) g_{T} g_{R}}
\end{array}\right)
\end{aligned}
$$

\section{B. Practical implementations of pseudo-rotation compensator}

Unfortunately, the 4 means in (15) and (16) are unknown in practice. With the proposed compensator, the estimation of the I/Q imbalance compensator $\mathbf{B}_{d}$ mainly depends on the estimation of the pseudo-rotation matrix $\mathbf{R}_{p}$. Indeed, the gain matrix $\mathbf{R}_{g}$ can be easily obtained from (18) with a small simplification: $s(t) \in \mathcal{R}_{1}$ is being replaced by $z(t) \in \mathcal{R}_{1}$.

Therefore, two indirect methods are proposed to compute the pseudo-rotation matrix $\mathbf{R}_{p}$. The first one is based on recursive pseudo-rotation while the second one corresponds to a low complexity version.

1) Recursive pseudo-rotation (RPR): Instead of computing the pseudo-rotation matrix angles (using (15) and (16)) from the 4 regions defined in Table I, the 4 means are computed from the imbalanced signal such as:

$$
\mathrm{E}\left[y_{I}(t)\right]_{l}=\mathrm{E}\left[y_{I}(t) \mid y(t) \in \mathcal{R}_{l}\right],
$$

where the region $\mathcal{R}_{l}, l \in\{1,2,3,4\}$, are defined in Table II. The implemented boundaries are also shown in Fig. 2 .

To achieve a high level of compensation, an iterative process is performed and detailed in Fig. 3. The pseudorotation matrix $\mathbf{R}_{p}$ is recursively computed and the process is stopped when the pseudo-rotation matrix $\mathbf{R}_{p t}$ at iteration $t$ is equal to the identity matrix with a precision $\epsilon$, i.e. 


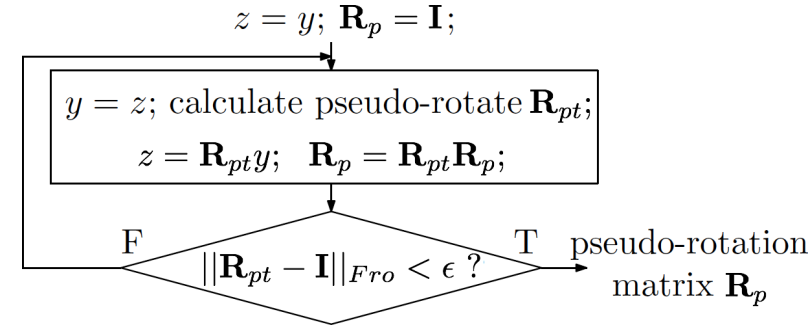

Fig. 3: Recursive method for pseudo-rotation computation.

$\left\|\mathbf{R}_{p t}-\mathbf{I} \mid\right\|_{F r o}<\epsilon$ where $\|.\|_{F r o}$ is the Frobenius norm [13]. We denote $N$ the resulting number of iterations.

2) Low complexity recursive pseudo-rotation (LRPR): As the complexity (in number of multiplications and additions) of RPR based compensator is quite important, the LRPR method is proposed to reduce it. To this aim, the effect of the number of iterations is first discussed. Fig. 4 shows the Frobenius norm $\left\|\mathbf{R}_{p t}-\mathbf{I}\right\|_{\text {Fro }}$ as a function of $E_{b} / N_{0}$ for different number of iterations $N$. With $N=5$ iterations, the precision $\epsilon$ is less than $10^{-6}$ at $E_{b} / N_{0}$ of $21.5 \mathrm{~dB}$ which gives a Symbol Error Rate (SER) of $10^{-3}$.

Fig. 5 shows the required number of iterations $N$ to achieve the Frobenius norm lower than $10^{-4}$ versus the TX phase imbalance. The figure also shows the relative ratio $d_{S E R}=\frac{(S E R)_{\text {ideal }}-(S E R)_{R P R}}{(S E R)_{\text {ideal }}}$, which gives the relative SER difference between the ideal (defined by (5)) and the RPR compensators. This figure shows that, for low TX phase imbalances, the minimum number of iterations to get performance close to the ideal compensator is about 5 . This number of iterations is given for a 256-QAM, it will be smaller for low modulation orders.

As presented above, the RPR compensator uses low order statistics to estimate the I/Q imbalance compensator. Thus, the size of necessary data for the convergence of the algorithm should be evaluated. Fig. 6 shows the power penalty versus

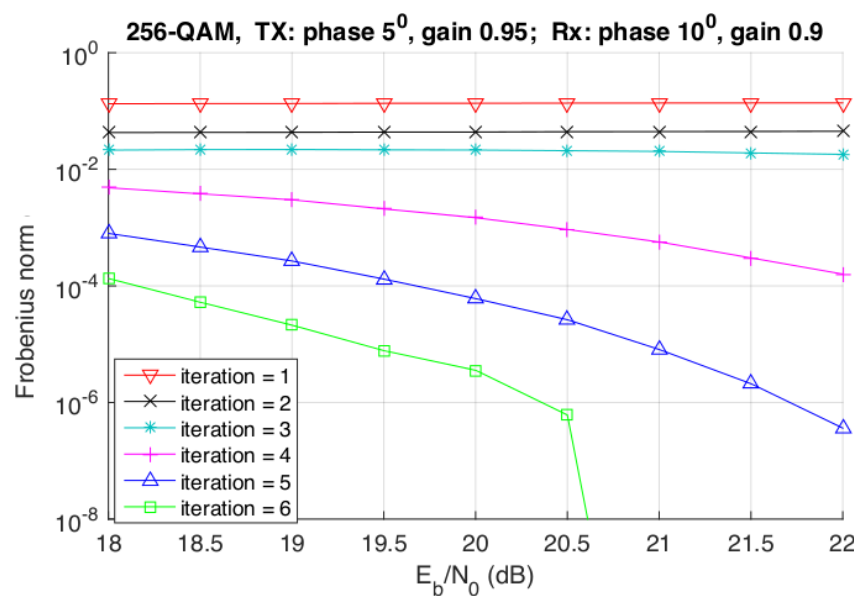

Fig. 4: Number of iterations $N$ required in the RPR.

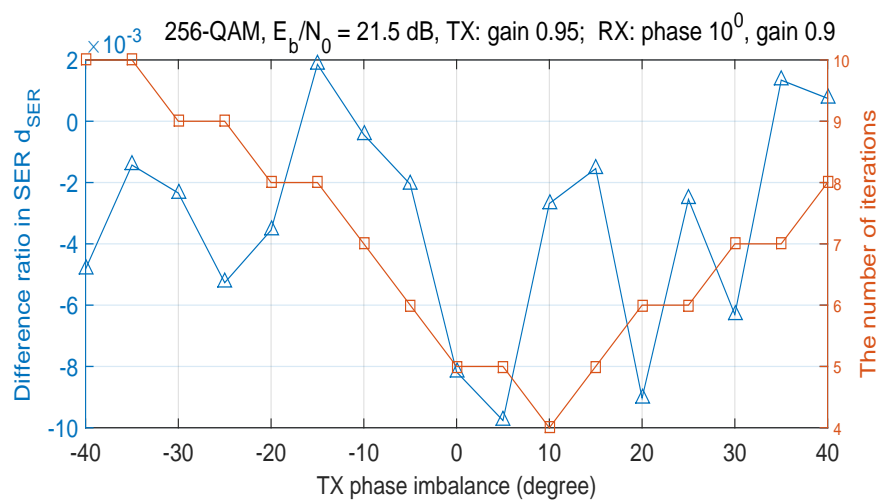

Fig. 5: Convergence of RPR compensator versus TX phase imbalance.

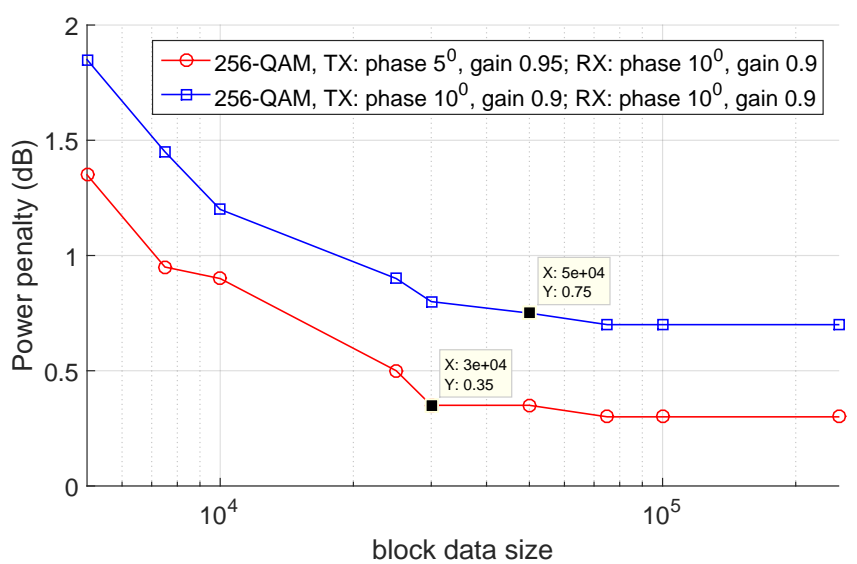

Fig. 6: Power penalty versus block data size $L$ of the RPR compensator with 5 iterations.

the data block size $L$ on which RPR is computed. The penalty is the difference in $E_{b} / N_{0}$ between the ideal and the RPR compensators to get a SER of $10^{-3}$. Simulations show that $L=3.10^{4}$ can ensure the statistical properties in this problem.

In addition to these practical choices of the number of iterations and the data block size, the complexity is also reduced using the 2-step algorithm shown in Fig. 7. First, an initialization step pre-estimates the pseudo-rotation matrix with a limited data block size $P_{1}$ and a number of iterations $N$. Then, a tracking phase updates the computation using only one iteration and a small data block size $P_{2}$ with $P_{2}<<P_{1}$. This implementation is called the low-complexity recursive pseudo-rotation (LRPR) method. Based on simulation results, LRPR is implemented with $N$ set to $5, P_{1}$ set to $3.10^{4}$ and $P_{2}$ set to $10^{3}$.

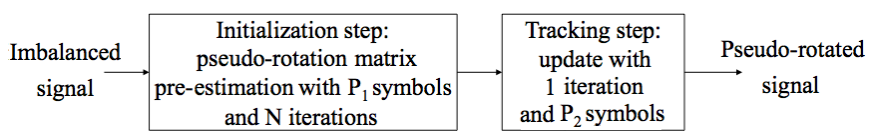

Fig. 7: LRPR block diagram. 


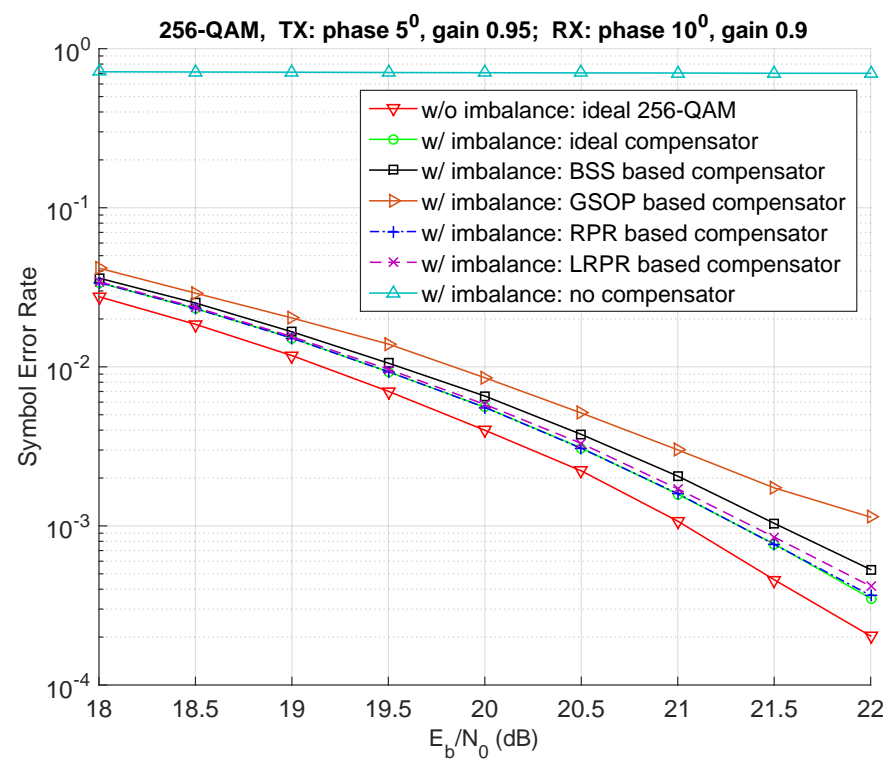

Fig. 8: System performance: SER versus $E_{b} / N_{0}$.

\section{Performance Results}

\section{A. SER performance}

The performance of several compensators are given on Fig. 8 that gives the SER as a function of $E_{b} / N_{0}$. RPR is implemented with $\epsilon=10^{-6}$ and $L=10^{6}$ in order to observe the best performance without taking into account the complexity. LRPR is implemented as mentioned before. The two practical implementations RPR and LRPR are compared with GSOP and BSS compensators. The performance of the ideal compensator (defined by (5)) is also shown. A 256QAM is used and the I/Q imbalance parameters are $\varphi_{T}=5^{\circ}$, $g_{T}=0.95, \varphi_{R}=10^{\circ}$ and $g_{T}=0.9$.

First, the figure shows that without compensation, the SER is so high that the transmission will not work and thus compensation is needed. By using the RPR compensator, the SER curve matches the ideal case. Furthermore, RPR outperforms the two other compensators with a penalty at SER of $10^{-3}$ of $0.25 \mathrm{~dB}$ and $0.75 \mathrm{~dB}$ compared with BSS and GSOP respectively.

Looking at the performance of the LRPR compensator, although it does not achieve the performance of ideal case, it also outperforms BSS and GSOP compensators. Comparing with the results in Fig. 6, the use of the tracking phase in LRPR allows an important decrease of the power penalty.

\section{B. Experimental validation using an optical communication testbed}

Fig. 9(a) shows the experimental setup [14] of a 10 Gbaud optical QPSK coherent system. To simplify the digital signal processing, the same laser is used at both $\mathrm{TX}$ and $\mathrm{RX}$ sides (acted as a local oscillator LO). At TX side, an Arbitrary Waveform Generator (AWG) generates two Pseudo-Random Binary Sequences (PRBSs) at the $10 \mathrm{GSymbol} / \mathrm{s}$ data rate. These PRBSs are used to pilot the two arms of a dual drive I/Q

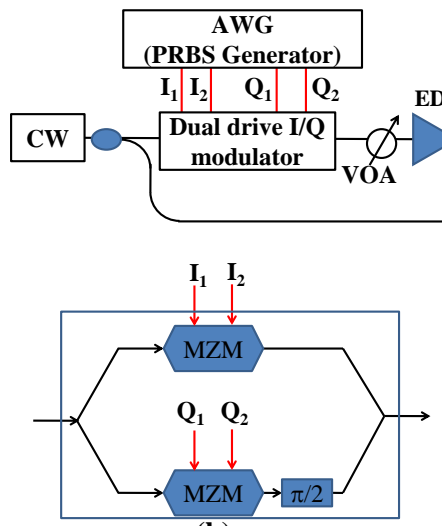

(b) (a)

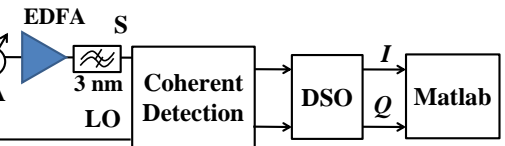

Fig. 9: Optical communication testbed. (a) The experimental testbed. (b) The I/Q modulator. (c) The coherent receiver.

modulator shown in Fig. 9(b), resulting in a 10 Gbaud QPSK signal. The I/Q modulator is composed of a Mach-Zehnder Modulator (MZM) in each arm and a phase shifter to form a $90^{\circ}$ phase difference between the two arms. By modifying the bias voltage that controls this phase difference, we can manually adjust the TX phase imbalance. In the setup chain, a Variable Optical Attenuator (VOA) is used to vary the received power. The signal is then amplified by an Erbium-Doped Fiber Amplifier (EDFA) cascaded to a 3-nm optical bandpass filter. At RX side, the optical QPSK signal is combined with the local oscillator in a QPSK integrated coherent receiver.

Fig. 9(c) presents the structure of the coherent receiver, in which the signal and the LO are split and cross-combined with the $90^{\circ}$ phase shift between the two branches. By this way, the resulting $\mathrm{I}$ and $\mathrm{Q}$ components after the balanced photo-detection can be achieved. These components are then digitized by a Digital Sampling Oscilloscope (DSO) with a $16 \mathrm{GHz}$ bandwidth at a sampling rate of $40 \mathrm{GSymbol} / \mathrm{s}$. The acquired data are post-processed off-line using Matlab environment. The received signal is processed through the pseudo-rotation compensator (RPR or LRPR) first and then a $T / 4$-fractionally spaced equalizer [15] is applied to eliminate the ISI introduced during the transmission processes ( $T$ being the symbol duration).

Fig. 10(a) shows the constellation of the received signal with a TX phase imbalance of $10^{\circ}$ and an Optical Signal to Noise Ratio (OSNR) of 25 dB. Fig. 10(b), Fig. 10(c) and Fig. 10(d) show the constellations when no compensator, RPR compensator and LRPR compensator are used respectively. All cases use the same $T / 4$-fractionally spaced equalizer.

The noise in Fig. 10(b) is higher than in the other ones, it means that the I/Q imbalance disturbs the operation of the equalizer. Fig. 10(c) and Fig. 10(d) shows that the pseudorotation technique has compensated I/Q imbalance even if the signal has ISI.

\section{CONClusion}

In this paper, the I/Q imbalance sensitivity of an M-QAM optical coherent system has been thoroughly analyzed through 

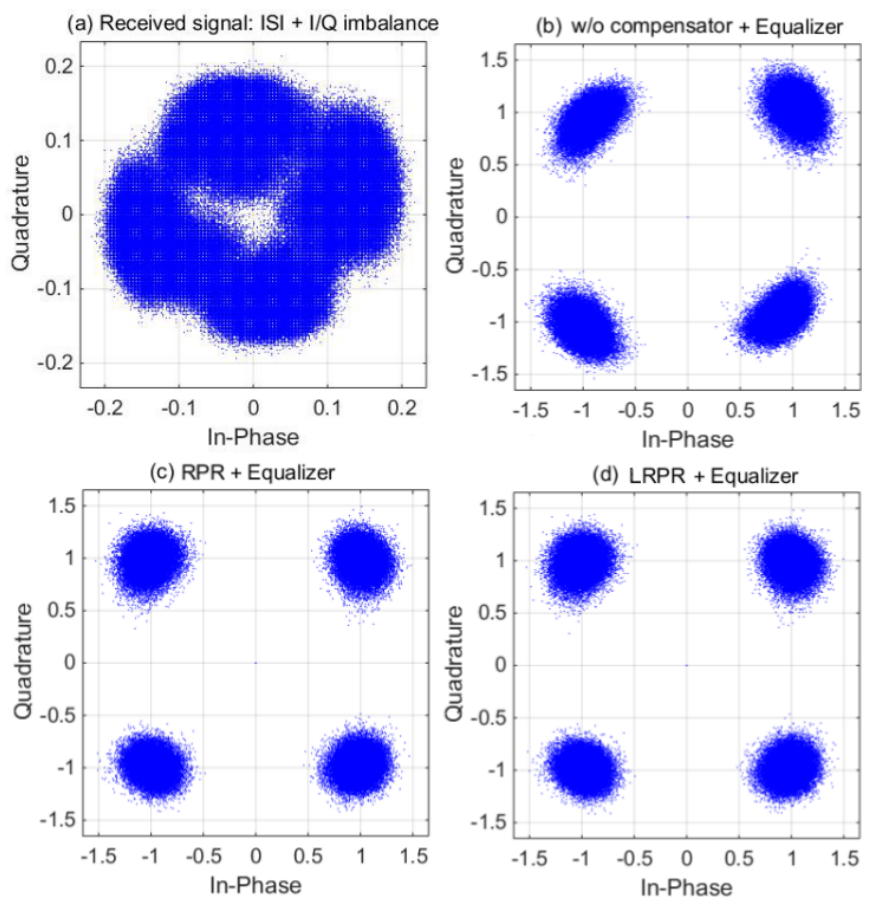

Fig. 10: I/Q imbalance and ISI on QPSK constellation.

error probability derivations, SER simulations and complexity evaluation. A new approach has been proposed using the pseudo-rotation technique. Through Monte Carlo simulations, it is shown that the proposed technique can achieve the same performance as the ideal compensator. The pseudo-rotation based compensator is a generic approach, so it can suit to low and high modulation orders and to small and large levels of imbalance. The experimental validation using an optical communication testbed shows that the behavior of the pseudorotation compensator is not affected by the presence of ISI. Future works will evaluate the performance of the compensator when the I/Q imbalance is combined with other factors such as phase noise and fading channel.

\section{REFERENCES}

[1] P. C. Schindler and et al., "Monolithic GaAs electro-optic IQ modulator demonstrated at $150 \mathrm{Gbit} / \mathrm{s}$ with 64QAM," Journal of Lightwave
Technology, vol. 32, no. 4, pp. 760-765, February 2014.

[2] M. Valkama, M. Renfors, and V. Koivunen, "Advanced methods for I/Q imbalance compensation in communication receivers," IEEE Transactions on Signal Processing, vol. 49, no. 10, pp. 2335 - 2344, October 2001.

[3] W. Chung, "Transmitter IQ mismatch compensation in coherent optical OFDM systems using pilot signals," Optics Express, vol. 18, no. 20, pp. $21308-21314,2010$.

[4] J. Lee, Y. Ha, B.-S. Shin, S. Kim, and B.-M. Kim, "Novel pilot scheme for transmitter IQ mismatch compensation in CO-OFDM system," IEEE Photonics Technology Letters, vol. 24, no. 17, pp. 1543-1545, September 2012.

[5] M. Gautier, P.-F. Morlat, and G. Villemaud, "IQ Imbalance Reduction in a SMI Multi-Antenna Receiver by Using a Code Multiplexing FrontEnd," in IEEE Vehicular Technology Conference (VTC-Spring 09), Barcelona, Spain, April 2009, pp. 1-5.

[6] W. Nam, H. Roh, J. Lee, and I. Kang, "Blind adaptive I/Q imbalance compensation algorithms for direct-conversion receivers," IEEE Signal Processing Letters, vol. 19, no. 8, pp. 475 - 478, August 2012.

[7] Z. Zhu, X. Huang, and H. Leung, "Joint I/Q mismatch and distortion compensation in direct conversion transmitters," IEEE Transactions on Wireless Communications, vol. 12, no. 6, pp. 2941 - 2951, June 2013.

[8] I. Fatadin, S. J. Savory, and D. Ives, "Compensation of quadrature imbalance in an optical QPSK coherent receiver," IEEE Photonics Technology Letters, vol. 20, no. 20, pp. 1733 - 1735, October 2008.

[9] J.-F. Cardoso, "Equivariant adaptive source separation," IEEE Transactions on Signal Processing, vol. 14, no. 12, pp. 3017 - 3030, December 1996.

[10] M. Valkama, M. Renfors, and V. Koivunen, "Blind signal estimation in conjugate signal models with application to I/Q imbalance compensation," IEEE Signal Processing Letters, vol. 12, no. 11, pp. 733 - 736, October 2005.

[11] M. Windisch, "Estimation and compensation of $I / Q$ imbalance in broadband communications receivers," Ph.D. dissertation, Dresden University of Technology, 2007.

[12] A. Metref, D. L. Guennec, and J. Palicot, "A Carrier Recovery loop for Cognitive Radio Applications," Journal on Circuits, Systems and Signal Processing (CSSP), vol. 20, no. 4, pp. 847-870, 2011.

[13] T. Ando and X. Zhan, "Norm inequalities related to operator monotone functions," Mathematische Annalen, vol. 315, no. 4, pp. 771-780, January 1999.

[14] T.-H. Nguyen, P. Scalart, M. Joindot, M. Gay, L. Bramerie, and C. Peucheret, "Joint simple blind IQ imbalance compensation and adaptive equalization for 16-qam optical communications," in IEEE International Conference on Communications, June 2015, pp. 49134918.

[15] I. Fijalkow, A. Touzni, and J. R. Treichler, "Fractionally Spaced Equalization Using CMA: Robustness to Channel Noise and Lack of Disparity," IEEE Transactions on Signal Processing, vol. 45, no. 1, pp. 56 - 66, January 1997. 